\title{
Embedding solenoids
}

by

\begin{abstract}
Alex Clark (Denton, TX) and Robbert Fokkink (Delft)
\end{abstract}
\begin{abstract}
A generalized solenoid is an inverse limit space with bonding maps that are (regular) covering maps of closed compact manifolds. We study the embedding properties of solenoids in linear space and in foliations.
\end{abstract}

A compact and connected topological space is called a continuum. A space $X$ is homogeneous if for every $x, y \in X$ there exists a homeomorphism $h: X \rightarrow X$ such that $h(x)=y$. The space is bihomogeneous if for every $x, y \in X$ there exists a homeomorphism that switches $x$ and $y$, i.e., $h(x)=y$ and $h(y)=x$. Settling an old problem of Knaster, K. Kuperberg [12] showed that there exist continua that are homogeneous but not bihomogeneous.

We show that there exist homogeneous continua in $\mathbb{R}^{4}$ that are not bihomogeneous. It is unknown whether there exist such continua in $\mathbb{R}^{k}$ for some $k<4$. The examples we provide are higher-dimensional analogues of the standard dyadic solenoid. We refer to these spaces as (generalized) solenoids.

First we study embeddings of generalized solenoids in linear space. This turns out to be related to the study of embeddings of manifolds. Next we study embeddings of solenoids in foliated bundles. In the final part of our paper we generalize to higher dimensions a dynamical characterization of one-dimensional solenoids by Thomas. We also show that a conjecture by Oversteegen on a possible topological characterization of solenoids turns out to be related to an old conjecture of Williams on expanding attractors, that was settled by Farrell and Jones [6].

\section{Solenoids}

Definition 1. A solenoid is an inverse limit space $\lim _{(}\left(M_{n}, p_{n}\right)$ such that the factor spaces $M_{n}$ are closed manifolds and the bonding maps $p_{n}: M_{n+1} \rightarrow M_{n}$ are covering maps that are not homeomorphisms.

2000 Mathematics Subject Classification: 37B45, 57N35.

Key words and phrases: attractor, continuum, foliated bundle, isolated minimal set, polynomial covering map, topological embedding. 
This generalizes the original definition of $P$-adic solenoids by van Dantzig and van der Waerden [5]. Higher-dimensional solenoids were first studied by McCord and by Schori $[14,20]$. Schori [20] has shown that generalized solenoids need not be topologically homogeneous. McCord [14] has shown that a generalized solenoid is homogeneous if all compositions of the bonding maps are regular covering maps. In this case, the solenoid is a principal bundle over a manifold with a structure group that is profinite, i.e., an inverse limit of finite groups (such groups are sometimes called Cantor groups). We have shown that if a solenoid is bihomogeneous, then its structure group contains an open abelian subgroup [4].

All solenoids under consideration in this paper are homogeneous. The results in [7] imply that this is equivalent to the condition that they are principal bundles with profinite structure groups.

The archetype of a solenoid is the dyadic solenoid, defined as the inverse limit over circles $S^{1}=\{z \in \mathbb{C}:|z|=1\}$ with bonding map $z \mapsto z^{2}$. It is an indecomposable continuum as well as a compact group and a hyperbolic attractor. The dyadic solenoid can be represented as the suspension over the adding machine. The adding machine can be extended to a homeomorphism $h: \mathbb{R}^{2} \rightarrow \mathbb{R}^{2}$, so the dyadic solenoid embeds as an invariant set of a flow on a toral domain in $\mathbb{R}^{3}$. Thomas [21] showed that the dyadic solenoid cannot occur as an isolated minimal set of a flow in $\mathbb{R}^{3}$, but Allaud and Thomas [2] showed that it can occur as an isolated minimal set of a flow in $\mathbb{R}^{4}$.

2. Embedding solenoids in euclidean space. We shall say that a solenoid is orientable if all factor spaces in the inverse limit system are orientable. Theorem 7 gives a criterion that is both necessary and sufficient for embedding a solenoid $\mathcal{S}$ that fibers over a manifold $M$ into $M \times \mathbb{R}^{2}$.

Lemma 2. An orientable $n$-dimensional solenoid does not embed in an orientable $(n+1)$-dimensional manifold.

Proof. This cohomology argument is similar to one in [21]. By compactification and by doubling, we may assume that the manifold $M$ is compact and closed. For an orientable $n$-dimensional solenoid $\mathcal{S}$, the Čechcohomology group with integer coefficients $\check{H}^{n}(\mathcal{S})$ is isomorphic to the direct $\operatorname{limit} \underset{\lim }{\longrightarrow}\left(\mathbb{Z}, p_{i j}^{*}\right)$. The homomorphism $p_{i j}^{*}: \mathbb{Z} \rightarrow \mathbb{Z}$ is given by $n \mapsto k n$ with $k$ equal to the degree of the covering $p_{i j}: S_{i} \rightarrow S_{j}$. In particular, $\check{H}^{n}(\mathcal{S})$ is isomorphic to the group of $P$-adic rationals for some prime sequence $P$. Then each $x$ in $\check{H}^{n}(\mathcal{S})$ has infinite height, meaning that there is a sequence of integers $n_{i} \rightarrow \infty$ and a sequence of elements $x_{i} \in \check{H}^{n}(\mathcal{S})$ satisfying $x=n_{i} x_{i}$. By duality there is an isomorphism $\check{H}^{n+1}(M, \mathcal{S}) \approx H_{0}(M-\mathcal{S})$, and zero is the only element of infinite height in the latter group. In the 
exact sequence

$$
\cdots \rightarrow \check{H}^{n}(M) \rightarrow \check{H}^{n}(\mathcal{S}) \rightarrow \check{H}^{n+1}(M, \mathcal{S}) \rightarrow \cdots
$$

all elements of $\check{H}^{n}(\mathcal{S})$ map to zero in $\check{H}^{n+1}(M, \mathcal{S})$ since the homomorphic image of an element of infinite height has infinite height. By exactness $\check{H}^{n}(M)$ must then map onto $\check{H}^{n}(\mathcal{S})$, which is impossible since $\check{H}^{n}(M)$ is finitely generated and $\check{H}^{n}(\mathcal{S})$ is not.

There exist two-dimensional orientable solenoids that are homogeneous but not bihomogeneous [4]. It is not possible to embed such solenoids in $\mathbb{R}^{3}$ by Lemma 2 , so we study if it is possible to embed them in $\mathbb{R}^{4}$. Our archetype, the dyadic solenoid, embeds in $\mathbb{R}^{3}$ through an embedding in $S^{1} \times \mathbb{R}^{2}$. We study if a two-dimensional solenoid, being a bundle over a surface $S$, embeds in $S \times \mathbb{R}^{2}$, which in its turn embeds in $\mathbb{R}^{4}$. This problem has been well studied for finite bundles, i.e., covering spaces.

Definition 3. Suppose that $M, N$ are manifolds. A covering map $p: M \rightarrow N$ is called polynomial if there exists a fiber-separating map $f: M \rightarrow \mathbb{R}^{2}$ for which $f(x) \neq f(y)$ if $p(x)=p(y)$ and $x \neq y$.

The name polynomial is derived from the fact that $M$ can be described as the set of zeroes of a family of polynomials parametrized by $N$ with non-zero discriminant. For a covering map $p: M \rightarrow N$ of degree $k$, the monodromy action on a fiber induces a characteristic homomorphism $\chi: \pi_{1}(N) \rightarrow S_{k}$, which is unique up to conjugation. The braid group $B_{k}$ on $k$ elements maps homomorphically onto the symmetric group $S_{k}$ by the forgetful map $b_{k}: B_{k} \rightarrow S_{k}$. A proof of the following theorem can be found in the beautiful little monograph [9].

THEOREM 4 (Hansen). A covering $p: M \rightarrow N$ is polynomial if and only if the characteristic homomorphism $\chi: \pi_{1}(N) \rightarrow S_{k}$ extends to a homomorphism $\widetilde{\chi}: \pi_{1}(N) \rightarrow B_{k}$ such that $b_{k} \circ \tilde{\chi}=\chi$.

This is a very nice algebraic characterization, but it is hard to verify by the complexity of the braid group. It is apparently unknown whether all coverings of orientable surfaces are polynomial. The best result in this direction is as follows.

Theorem 5 (Petersen, [18]). A regular covering $p: M \rightarrow N$ of a closed manifold with free abelian first homology $H_{1}(N)$ is polynomial if the quotient group $\pi_{1}(N) / \pi_{1}(M)$ is solvable.

Here and in what follows, for a covering map of manifolds $p: M \rightarrow N$ we identify $\pi_{1}(M)$ with the subgroup $p_{*}\left(\pi_{1}(M)\right)$ of $\pi_{1}(N)$.

It turns out that we need to adapt the definition of polynomial coverings somewhat to give a necessary and sufficient condition on solenoids that fiber through the trivial bundle. 
Definition 6. Suppose that $M_{i}$ are manifolds for $i=0,1,2$ and that $p_{2}: M_{2} \rightarrow M_{1}$ and $p_{1}: M_{1} \rightarrow M_{0}$ are covering maps. We say that $p_{1}$ is polynomial with respect to $p_{2}$ if there exists a map $f: M_{2} \rightarrow \mathbb{R}^{2}$ such that such that $f(x) \neq f(y)$ if $p_{2}(x) \neq p_{2}(y)$ and $p_{1} \circ p_{2}(x)=p_{1} \circ p_{2}(y)$, and we say that the map $f$ is fiber-separating with respect to $\left\langle p_{1}, p_{2}\right\rangle$.

Note that we retrieve the definition of polynomial covering if $p_{2}$ is the identity. Also note that if $p_{1}$ is a polynomial covering, then for any covering $p_{2}$, it is polynomial with respect to $p_{2}$. We say that a solenoid $p: E \rightarrow S$ factors through the trivial bundle $S \times \mathbb{R}^{2}$ if there exists a fiber-separating map $f: E \rightarrow \mathbb{R}^{2}$ for which $f(x) \neq f(y)$ if $p(x)=p(y)$ and $x \neq y$.

THEOREM 7. Suppose that $M_{\infty}$ is a solenoid over a base space $M_{0}$. Then $M_{\infty}$ factors through $M_{0} \times \mathbb{R}^{2}$ if and only if it can be represented as an inverse limit $M_{\infty}=\lim _{(}\left(M_{i}, p_{i}\right)$ such that $p_{i-1}$ is polynomial with respect to $p_{i}$ for all $i>1$.

Proof. First suppose that $M_{\infty}=\varliminf_{(m}\left(M_{i}, p_{i}\right)$ and that the covering maps $p_{i-1}$ are polynomial with respect to $p_{i}$ for all $i$. There exist $f_{i}: M_{i+1} \rightarrow$ $\mathbb{R}^{2}$ that are fiber-separating with respect to $\left\langle p_{i-1}, p_{i}\right\rangle$ and by compactness, we may assume that $\left|f_{i}(x)\right| \leq 1$ for all $x \in M_{i}$. By compactness of the fibers, there exist $\varepsilon_{i}>0$ such that $\left|f_{i}(x)-f_{i}(y)\right|>\varepsilon_{i}$ if $p_{i}(x) \neq p_{i}(y)$ and $p_{i-1} \circ p_{i}(x)=p_{i-1} \circ p_{i}(y)$. Without loss of generality we may assume that $\sum_{i=k+1}^{\infty} \varepsilon_{i}<\varepsilon_{k}^{2}$ and that $\varepsilon_{k}<1$ for all $k \geq 1$. Define $F_{i}: \underset{\lim }{\longleftarrow}\left(M_{i}, p_{i}\right) \rightarrow$ $\mathbb{R}^{2}$ as the composition of the projection onto the $i$ th coordinate and the map $f_{i}$. Then the infinite sum $F=\sum_{i=1}^{\infty} \varepsilon_{i} F_{i}$ is well defined and we verify that $F: M_{\infty} \rightarrow \mathbb{R}^{2}$ is fiber-separating. Indeed, suppose that $F(x)=F(y)$ for $x, y \in M_{\infty}$ that are in the same fiber over $M_{0}$. These points can be represented by sequences $x_{i}, y_{i}$ in the inverse limit. Let $i$ be the first index for which $x_{i-1} \neq y_{i-1}$. Then $\left|f_{i}\left(x_{i}\right)-f_{i}\left(y_{i}\right)\right|>\varepsilon_{i}$ while $f_{j}\left(x_{j}\right)=f_{j}\left(y_{j}\right)$ for all $j<i$. By the triangle inequality and by the condition on the $\varepsilon_{i}$ above, we see that $F(x) \neq F(y)$. Hence $F: M_{\infty} \rightarrow \mathbb{R}^{2}$ separates the fibers of $\pi_{0}$, and $x \mapsto\left(\pi_{0}(x), F(x)\right)$ yields the desired factorization through $M_{0} \times \mathbb{R}^{2}$.

Conversely, suppose that the solenoid $\pi_{0}: M_{\infty} \rightarrow M_{0}$, which is represented as an inverse limit $\lim _{(}\left(M_{n}, p_{n}\right)$, admits a fiber-separating map $f: M_{\infty} \rightarrow \mathbb{R}^{2}$. By Tietze's extension theorem, this fiber-separating map extends to $f: \prod M_{i} \rightarrow \mathbb{R}^{2}$. We fix a base point $m_{n} \in M_{\infty} \subset \prod M_{n}$ and define the embedding $e_{n}: M_{n} \rightarrow \prod M_{n}$ by mapping $x \in M_{n}$ onto a sequence $x_{i}$ which has its tail derived from the base point and its head derived from $x$. To be more precise, $x_{n}=x$ and $x_{j}=p_{j} \circ \cdots \circ p_{n-1}(x)$ if $j<n$ and $x_{j}=m_{j}$ if $j>i$. The covering map $p_{j i}=p_{j} \circ \cdots \circ p_{i-1}: M_{i} \rightarrow M_{j}$ is represented by the composition of $e_{i}$ and $\pi_{j}$. We argue by contradiction that for each $j$ there exists an $i>j$ such that $f \circ e_{i}$ is fiber-separating with respect to $\left\langle p_{j}, p_{j i}\right\rangle$. Suppose that for each $k>j$ there exist $x_{k}, y_{k} \in M_{k}$ 
with $p_{j k}\left(e_{k}\left(x_{k}\right)\right) \neq p_{j k}\left(e_{k}\left(y_{k}\right)\right)$ while $p_{j} \circ p_{j k}\left(e_{k}\left(x_{k}\right)\right)=p_{j} \circ p_{j k}\left(e_{k}\left(y_{k}\right)\right)$ and $f\left(e_{k}\left(x_{k}\right)\right)=f\left(e_{k}\left(y_{k}\right)\right)$. By compactness, there exist limit points $x_{\infty}, y_{\infty}$, which are necessarily contained in $M_{\infty}$, such that $x_{\infty}, y_{\infty}$ have equal $(j-1)$ th coordinate and unequal $j$ th coordinate, while $f\left(x_{\infty}\right)=f\left(y_{\infty}\right)$. This contradicts the assumption that $f$ is fiber-separating. So there exists an $i>j$ such that $p_{j}$ is polynomial with respect to $p_{j i}$. By deleting all indices in between $j$ and $i$ we can construct an inverse limit representation of the solenoid such that all coverings $p_{i}$ are polynomial with respect to $p_{i+1}$.

Corollary 8. An orientable two-dimensional solenoid $\underset{\lim }{\longleftarrow}\left(S_{i}, p_{i}\right)$ embeds in $\mathbb{R}^{4}$ if all covering maps are polynomial.

Proof. According to Theorem 7 the solenoid embeds in $S_{0} \times \mathbb{R}^{2}$. Since $S_{0} \times \mathbb{R}$ is homeomorphic to a tubular neighborhood of $S_{0}$ in $\mathbb{R}^{3}$, the solenoid embeds in $\mathbb{R}^{4}$.

Our next corollary is the result that we aimed for in the first place.

COROLlary 9. There exist homogeneous non-bihomogeneous continua in $\mathbb{R}^{4}$.

Proof. The fundamental group of an orientable surface of genus 2 can be represented as $G=\left\langle x_{1}, x_{2}, x_{3}, x_{4}:\left[x_{1}, x_{2}\right]\left[x_{3}, x_{4}\right]\right\rangle$. The subgroup $F \subset G$ generated by $x_{1}$ and $x_{3}$ is free. Let $N_{0} \supset N_{1} \supset N_{2} \supset \cdots$ be a descending chain of normal subgroups of $F$ with finite index and with intersection $\{e\}=$ $\bigcap N_{i}$. Define the homomorphism $f: G \rightarrow F$ by $f\left(x_{1}\right)=x_{1}, f\left(x_{2}\right)=e$, $f\left(x_{3}\right)=x_{3}, f\left(x_{4}\right)=e$. Then $f^{-1}\left(N_{i}\right)$ is a normal subgroup of $G$ of finite index, and the action of $G$ on $G / f^{-1}\left(N_{i}\right)$ is isomorphic to the action of $F$ on $F / N_{i}$. The $N_{i}$ are free groups since they are subgroups of a free group. Therefore the action of $N_{i}$ on $N_{i} / N_{i-1}$ factors through the braid group and so does the pull back of this action to $G$. It follows by Hansen's criterion that the covering maps that correspond to $f^{-1}\left(N_{i}\right)$ are polynomial. The inverse limit of the covering maps gives a homogeneous solenoid that embeds in $\mathbb{R}^{4}$. The profinite completion $\lim F / N_{i}$ contains $F$ as a dense subgroup, so it does not contain an open abelian subgroup. The structure group of the solenoid is isomorphic to $\lim F / N_{i}$, so it is not bihomogeneous.

COROLlary 10. A bihomogeneous orientable two-dimensional solenoid embeds in $\mathbb{R}^{4}$. More generally, any bihomogeneous solenoid that fibers over a manifold $M_{0}$ with free abelian first homology embeds in $M_{0} \times \mathbb{R}^{2}$.

Proof. A bihomogeneous solenoid can be represented as an inverse $\operatorname{limit} \lim \left(M_{i}, p_{i}\right)$ with an abelian structure group $\underset{\lim }{\longleftarrow}\left(\pi_{1}\left(M_{0}\right) / \pi_{1}\left(M_{i}\right)\right)$. By Petersen's result, all coverings are polynomial so the solenoid embeds in $M_{0} \times \mathbb{R}^{2}$. In the orientable two-dimensional case, $M_{0}$ can be chosen to be an orientable surface $S_{0}$. 
This last result is a generalization of a result in [11] on the embedding of torus-like solenoids.

3. Embedding solenoids in foliated bundles. Solenoids have the local structure of minimal sets of foliations. Now that we have a criterion for embedding a solenoid in a trivial $\mathbb{R}^{2}$-bundle, we study whether this embedding extends to a foliation of the bundle that is transverse to the fibers. Theorem 14 gives a criterion that is both necessary and sufficient for embedding a solenoid $\mathcal{S}$ that fibers over a manifold $M$ into a foliated bundle with total space $M \times \mathbb{R}^{2}$.

Definition 11. A foliated bundle consists of a bundle $p: E \rightarrow B$ and a foliation $\mathcal{F}$ of $E$ transverse to the fibers.

We shall assume that the leaves of $\mathcal{F}$ project onto the base space, in which case the bundle is called flat, and that it is a vector bundle. The fundamental group of the base space acts on a fiber, as follows. Fix a base point $b \in B$ and let $x \in F_{b}$ be an element of the fiber. A closed path $w$ based at $b$ lifts to a unique path $\widetilde{w}$ in $\mathcal{F}$ that has end point $x$ and initial point in $F_{b}$. The holonomy action associates $x$ to the initial point of the path of $\widetilde{w}$ and thus gives a representation $\pi_{1}(B, b) \rightarrow \operatorname{Homeo}\left(F_{b}\right)$. It is known that the holonomy action alone allows a reconstruction of the bundle by means of a suspension [10, II.1.3.1].

For a (connected) manifold $M$ with universal covering space $\widetilde{M}$, we identify the fundamental group $\pi_{1}(M)$ with the group of deck transformations of $\widetilde{M}$, with paths $\gamma$ acting on the right $x \mapsto x \cdot \gamma$. For a representation $\chi: \pi_{1}(M) \rightarrow \operatorname{Homeo}\left(\mathbb{R}^{k}\right)$, the suspension $\Sigma(\chi)$ is defined as the quotient space of $\widetilde{M} \times \mathbb{R}^{k}$ under the equivalence relation $(x, v) \sim\left(x \cdot \gamma, \chi(\gamma)^{-1} v\right)$. The trivial foliation by $\widetilde{M} \times\{v\}$ for a fixed $v \in \mathbb{R}^{k}$ projects onto a foliation of the suspension. The holonomy action on the suspension recovers the representation $\chi$. So in order to embed a solenoid in a suspension (flat bundle) we need to extend the holonomy action on the Cantor fiber $\mathcal{C}$ to $\mathbb{R}^{k}$, as summarized by the following lemma.

Lemma 12. A solenoid $M_{\infty}$ over a manifold $M_{0}$ embeds as a minimal set in a flat $\mathbb{R}^{k}$-bundle over $M_{0}$ if and only if its holonomy action extends from $\mathcal{C}$ to $\mathbb{R}^{k}$.

We are interested in the case that $k=2$. The point remaining is now to decide when a flat $\mathbb{R}^{2}$-bundle, given by a representation $\pi_{1}\left(M_{0}\right) \rightarrow \mathbb{R}^{2}$, is in fact a trivial bundle, i.e., when the total space is homeomorphic to the product of the base space and the fiber via a homeomorphism preserving the fibers of the bundle. The suspension need not be a trivial bundle. The condition for triviality is as follows (see [10]). 
LEMma 13. Suppose that $M$ is a closed manifold with base point $m_{0}$ and that $\chi: \pi_{1}\left(M, m_{0}\right) \rightarrow \operatorname{Homeo}\left(\mathbb{R}^{k}\right)$ is a homomorphism. Then the suspension $\Sigma(\chi)$ is equivalent to a trivial bundle if and only if there exists a map $f: \widetilde{M} \rightarrow \operatorname{Homeo}\left(\mathbb{R}^{k}\right)$ such that $f(x \cdot \gamma)=f(x) \circ \chi(\gamma)$ for all $\gamma \in \pi_{1}(M)$.

Proof. Identify the deck transformations of the universal covering $\widetilde{M} \rightarrow$ $M$ with the fundamental group. By definition, the suspension is the quotient space of $\widetilde{M} \times \mathbb{R}^{k}$ under the equivalence $(x, v) \sim\left(x \cdot \gamma, \chi(\gamma)^{-1}(v)\right)$ for $\gamma \in \pi_{1}\left(M, m_{0}\right)$. The suspension is trivial if and only if there exists a map $f: \Sigma(\chi) \rightarrow \mathbb{R}^{k}$ which maps each fiber homeomorphically onto $\mathbb{R}^{k}$. Lift this map to $\widetilde{M} \times \mathbb{R}^{k}$ and by abuse of notation, for $x \in \widetilde{M}$ let $f(x)$ be the restriction of the lift to the fiber $\{x\} \times \mathbb{R}^{k} \rightarrow \mathbb{R}^{k}$. The condition $f(x \cdot \gamma)=f(x) \circ \chi(\gamma)$ follows as $f$ has to respect the equivalence.

Denote the fundamental group of $M$ by $G$. The universal covering of $M$ is a (right) $G$-space. The space $\operatorname{Homeo}\left(\mathbb{R}^{k}\right)$ is a right $G$-space as well under the action $H \cdot g=H \circ \chi(g)$. So this lemma says that $\Sigma(\chi)$ is trivial if and only if there exists a $G$-equivariant map $\widetilde{M} \rightarrow \operatorname{Homeo}\left(\mathbb{R}^{k}\right)$. We give a criterion that decides whether the presentation $\chi$ induces a trivial bundle and that does not depend on the universal covering space.

TheOREM 14. Suppose that $G \subset H$ are groups and that $X$ is a $G$-space subject to the following conditions:

(1) $H$ is a topological group that is locally simply connected and has trivial homotopy groups $\pi_{k}(H)$ for $k \geq 2$.

(2) $G=\left\langle x_{i}: R_{j}\left(x_{i}\right)\right\rangle$ is finitely presented.

(3) $G$ is the fundamental group of a closed triangulable manifold $M$ and $X$ is its universal covering space with the monodromy action.

Then there exists a G-equivariant map $f: X \rightarrow H$ if and only if there exist paths $x_{j}^{t}$ in $H$ connecting $e$ to $x_{j}$ such that the paths $t \mapsto R_{j}\left(x_{i}^{t}\right)$ are contractible for all $j$.

Proof. We first remark that the condition does not depend on the choice of the representation of $G$. Any other choice of generators and relations $\left\langle x_{i}^{\prime}: R_{j}^{\prime}\left(x_{i}^{\prime}\right)\right\rangle$ gives rise to a rewriting of $x_{i}^{\prime}$ in terms of $x_{i}$ and so it gives paths $x^{\prime t}{ }_{i}$ in terms of $x_{i}^{t}$. Rewriting a relation $R_{j}^{\prime}\left(x_{i}^{\prime}\right)$ in terms of $x_{i}$ then gives contractible paths.

To show the necessity of the condition, let $f: X \rightarrow H$ be a $G$-equivariant map. For an element $g \in G$ and an element $x \in X$ let $w$ be a path from $x$ to $x \cdot g$ in $X$. The homotopy class of $w$ is unique as $X$ is simply connected and therefore so is the homotopy class of the path $f(x)^{-1} f(w)$, which connects $e$ to $g$ in $H$. In particular, the homotopy class of this path does not depend on the choice of $w$. Since $H$ is locally simply connected, paths that are 
sufficiently close are homotopic. This implies that the homotopy class of $f(x)^{-1} f(w)$ does not depend on the choice of $x$ either. So for any $g \in G$ we have a unique homotopy class $w(g)$ of paths in $H$ connecting $g$ to $e$. For $g, h \in G$ we may combine a path from $x$ to $x \cdot g$ with a path from $x \cdot g$ to $x \cdot g h$. Without loss of generality, we may assume that $f(x)=e$, so the first homotopy class is $w(g)$ and the second is $g w(h)$. The path homotopy composition of $w(g)$ and $g w(h)$ is homotopic to $w(g) w(h)$, implying that $w(g h)=w(g) w(h)$. For a generator $x_{i}$ choose a path $x_{i}^{t}$ in the class $w\left(x_{i}\right)$. For a relation $R_{j}$, by definition the path $R_{j}\left(x_{i}^{t}\right)$ is in the class $R_{j}\left(w\left(x_{i}\right)\right)$. Since $w$ preserves multiplication, the class is the trivial one, so $R_{j}\left(x_{i}^{t}\right)$ is homotopic to the constant path. Hence the necessity of the condition.

We show the sufficiency of the condition, which follows from a standard obstruction argument. The manifold $M$ is finitely triangulable and the vertices of the triangulation can be contracted to a single point, giving a $C W$-decomposition of $M$ with a single vertex and a finite number of 1-cells, the homotopy classes $x_{i}$ of which generate the fundamental group $G$. Lift this $C W$-decomposition to a decomposition of the universal covering space $X$. The preimage of the single vertex is a $G$-orbit in $X$, from which we fix an element $v \in X$ that we map to $e \in H$. According to our condition, which does not depend on the choice of the generators, we can define a map on the 1-skeleton of our $C W$-decomposition of $X$ as follows. First extend to the preimages of the $x_{i}$ starting from $v$ by mapping these to the paths $x_{i}^{t} \in H$. Then extend $G$-equivariantly. The 2-cells of the decomposition of $M$ correspond to the relations of the fundamental group. Our condition allows an extension over the 2-skeleton of $X$ since the boundaries of the 2 -skeleton are mapped to contractible paths. We first extend to the cells that are attached to $v$ and then extend $G$-equivariantly. As $H$ has homotopy groups $\pi_{k}(H)=0$ for $k \geq 2$, it is possible to extend the map over the higher-dimensional cells.

This result applies to our problem, by the following deep result of Yagasaki, which builds on substantial previous work [25].

THEOREM 15. Homeo $\left(\mathbb{R}^{2}\right)$ is locally simply connected, and the path component of the identity has the homotopy type of a circle.

The result we aimed for now follows as a corollary.

COROLlaRY 16. There exists a homogeneous, non-bihomogeneous solenoid that embeds as a minimal set of a codimension-2 foliation of $S_{0} \times \mathbb{R}^{2}$, where $S_{0}$ is a closed orientable surface of genus 2 .

Proof. Take the solenoid of Corollary 9. Its holonomy action factors through an action of the free group $F_{2}$ on two elements. The action of $F_{2}$ on $\mathcal{C}$ extends to $\mathbb{R}^{2}$ since every homeomorphism on a Cantor set can be extended to an orientation preserving transformation of the plane [16]. The 
action of the fundamental group $\left\langle x_{1}, x_{2}, x_{3}, x_{4}:\left[x_{1}, x_{2}\right]\left[x_{3}, x_{4}\right]\right\rangle$ therefore extends to the plane, with $x_{i}$ acting as the identity for $i=2,4$. The paths $\gamma_{2}, \gamma_{4}$ in Theorem 14 can therefore be chosen to be constant. So this solenoid embeds as a minimal set in an $\mathbb{R}^{2}$-bundle over $S_{0}$ which is equivalent to the trivial bundle $S_{0} \times \mathbb{R}^{2}$.

Sacksteder [19] has given an example of an exceptional minimal set in a codimension-1 foliation, which fibers over a surface of genus 2 . The construction has some similarity with the example in our corollary above, though Sacksteder's minimal set is not a solenoid.

4. The dynamics of embedding solenoids in bundles. As we shall see, the following concept is closely related to the occurrence of solenoids in bundles.

Definition 17. A group action of $G$ on a metric space $X$ is equicontinuous if for any $\varepsilon>0$ there is a corresponding $\delta>0$ so that for any $g \in G$ we have

$$
d(g \cdot x, g \cdot y)<\varepsilon \quad \text { whenever } \quad d(x, y)<\delta .
$$

If $X$ is compact this notion does not depend on the choice of the metric. We say that an invariant set of $\Sigma(\chi)$ is equicontinuous if the holonomy action on a fiber is equicontinuous. This notion does not depend on the choice of the fiber. The following characterization of solenoids in terms of dynamical systems has been given by Thomas [21].

THEOREM 18. If an equicontinuous flow has a compact one-dimensional minimal set, then the minimal set is homeomorphic to a solenoid or the circle.

In the one-dimensional case, the return maps to a fiber (i.e., the holonomy maps) can be embedded in a flow on the total space. In the higherdimensional case, we cannot expect the holonomy maps on fibers to embed in a Lie group action on the total space. Nonetheless, Thomas's dynamic characterization of one-dimensional solenoids does generalize to foliated bundles.

If $S$ is a closed manifold, then as in the previous section $\Sigma(\chi)$ is the suspension of a homomorphism $\chi: \pi_{1}\left(S, s_{0}\right) \rightarrow \operatorname{Homeo}\left(\mathbb{R}^{k}\right)$. When $I$ is an invariant set of $\Sigma(\chi)$ then its intersection with a fiber $I_{0}=p^{-1}\left(s_{0}\right) \cap I$ is invariant under the holonomy action, so this induces a homomorphism $\chi_{I}: \pi_{1}\left(S, s_{0}\right) \rightarrow \operatorname{Homeo}\left(I_{0}\right)$ with $\chi_{I}(h)=\left.\chi(h)\right|_{I_{0}}$. When $\mathcal{S}$ is a solenoid and $\mathcal{S} \rightarrow S$ is a principal bundle map with Cantor fiber, we refer to this as a principal representation.

THEOREM 19. If a free $G$-action on a Cantor set $\mathcal{C}$ is minimal and equicontinuous, then $G$ compactifies to a group homeomorphic to $\mathcal{C}$. 
Proof. The special case of an infinite cyclic $G$ is a key lemma in [21]. For simplicity of this proof, we shall consider $G$ as acting on the left on $\mathcal{C}$. For a given $g \in G$, the action by $g, x \mapsto g . x$, determines a homeomorphism of $\mathcal{C}$. Then the mapping $e: G \rightarrow \operatorname{Homeo}(\mathcal{C})$ with $e(g)(x):=g \cdot x$ is a monomorphism by the freeness of the action. By the equicontinuity of the action of $G$, $e(G)$ is an equicontinuous subgroup of $\operatorname{Homeo}(\mathcal{C})$, which then has compact closure $\mathcal{G}$ in $\operatorname{Homeo}(\mathcal{C})$.

Fix an element $x \in \mathcal{C}$ and define the continuous mapping $h: \mathcal{G} \rightarrow \mathcal{C}$ by $h(g)=g(x)$. Given $y \in \mathcal{C}$, the minimality of the action of $G$ yields a sequence $\left\{g_{i}\right\} \subset e(G)$ with $g_{i}(x) \rightarrow y$. For a convergent subsequence $\left\{g_{i_{k}}\right\} \rightarrow g$ in $\mathcal{G}$, we then have $h(g)=g(x)=y$, demonstrating that $h$ is onto. Similarly, the freeness of the action of $G$ can be used to demonstrate that $h$ is one-to-one and hence a homeomorphism.

THEOREM 20. If $\mathcal{S}$ is an invariant solenoid in $\Sigma(\chi)$ for which $p: \mathcal{S} \rightarrow S$ is a principal representation, then $\mathcal{S}$ is equicontinuous. Conversely, if $\mathcal{S}$ is an equicontinuous minimal set of $\Sigma(\chi)$ of the same dimension as $S$, then $\mathcal{S}$ is a solenoid or a closed manifold.

Proof. If $p: \mathcal{S} \rightarrow S$ is a principal representation of the solenoid $\mathcal{S}$, consider the group $G$ of fiber preserving homeomorphisms of $\mathcal{S}$, i.e., $G$ is the Cantor structure group of the solenoid. The holonomy action gives a natural embedding $\chi_{I}\left(\pi_{1}\left(S, s_{0}\right)\right) \rightarrow G$, thus providing a compactification of $\chi_{I}\left(\pi_{1}\left(S, s_{0}\right)\right)$ in Homeo $\left(I_{0}\right)$. This yields the desired equicontinuity.

Suppose $\mathcal{S}$ is an equicontinuous minimal set of $\Sigma(\chi)$ and let $[x, s]$ denote the equivalence class of an element in $\widetilde{M} \times \mathbb{R}^{k}$. The holonomy action on a fiber

$$
\gamma:[x, v] \mapsto[x \cdot \gamma, v]
$$

extends to a map $\mathcal{S} \rightarrow \mathcal{S}$. This map has an inverse induced by the inverse holonomy element, and so the map is a homeomorphism. As in 19, it follows that this holonomy group compactifies to a Cantor group naturally homeomorphic to a fiber. Thus $\mathcal{S}$ is a principal bundle, hence a solenoid.

Recall that an attractor of a flow $\phi: X \times \mathbb{R} \rightarrow X$ is a non-empty, invariant, compact subset $A \subset X$ admitting a neighborhood $U$ such that for any given neighborhood $V$ of $A$ there is a $T$ such that $\phi(U \times[T, \infty)) \subset V$. In the setting of bundles this definition modifies as follows.

Definition 21. $A$ is an attractor of a suspension $\Sigma(\chi)$ if for some neighborhood $U$ of $A$ and for some set $\left\{g_{1}, \ldots, g_{k}\right\}$ of generators of $\pi_{1}\left(S, s_{0}\right)$ and for any neighborhood $V$ of $A$ there exists an $N$ so that for all $n \geq N$,

$$
g^{n} \cdot[x, v]=\left[x \cdot g^{n}, v\right]=\left[x, \chi\left(g^{-n}\right) v\right] \in V
$$

for any given $g \in\left\{g_{1}, \ldots, g_{k}\right\}$ and any $[x, v] \in U$. 
Thomas [21] showed that a (one-dimensional) solenoid cannot be an isolated set for a flow on a three-dimensional manifold. Ideally, this should generalize to the fact that a solenoid cannot be an isolated minimal set in a codimension- 2 foliation. We are only able to obtain the following weaker result, related to a shape-theoretic result in [8], that a solenoid cannot be an attractor for any foliation.

TheOREM 22. Any attractor $A$ of a suspension $\Sigma(\chi)$ over a closed orientable surface $S$ has the shape of a finite polyhedron.

Proof. The surface $S$ admits a branched covering of branching order 2 of the torus. This allows us to define a flow on $S$ that projects under such a covering onto a flow on the torus with periodic orbits, with the exception of two singular points and the orbits leading to and from these fixed points. One can then construct a finite number of flows $\left\{\phi_{1}, \ldots, \phi_{m}\right\}$ on $S$ so that each point of $S$ is contained in a neighborhood of points having orbits under one of the flows $\phi_{i}$ which are periodic with homotopy class from among the set of generators $\left\{x_{1}, \ldots, x_{2 k}\right\}$ of $\pi_{1}\left(S, s_{0}\right)$. Consider a nested sequence of compact neighborhoods $V_{n} \rightarrow \mathcal{S}$ with $V_{n} \subset \operatorname{Int}\left(V_{n-1}\right)$. Then for each $n$ there is a continuous map $f_{n}: \Sigma(\chi) \rightarrow[0,1]$ with $f_{n}^{-1}(0)=V_{n}$. For each $n$ there are then flows $\phi_{i}^{n}$ generated by the vector fields with vectors given by the vector fields for $\phi_{i}$ multiplied by the scalar $f_{n}$. By the hypothesis that $A$ is an attractor we can choose $T(n, i)>0$ so that

$$
R_{n}: U \times[0, T(n, 1)+\cdots+T(n, m)] \rightarrow V_{n}
$$

given by

$$
R_{n}(x, t)=\phi_{i}^{t-(T(n, 1)+\cdots+T(n, i-1))} \circ \phi_{i-1}^{T(n, i-1)} \circ \cdots \circ \phi_{1}^{T(n, 1)}(x)
$$

for $t \in[0, T(n, 1)+\cdots+T(n, i))$ is a strong deformation retraction of $U$ into $V_{n}$. Hence, $\mathcal{S}$ must have the shape of the manifold $U$.

5. Oversteegen's conjecture. Hagopian has characterized one-dimensional solenoids as the homogeneous indecomposable continua containing only arcs as proper subcontinua. There is another characterization of onedimensional solenoids.

Theorem 23 (Aarts et al., [1]). Denote the Cantor set by $\mathcal{C}$. A topological space $X$ is homeomorphic to a one-dimensional solenoid if and only if $X$ is a homogeneous continuum which has an open basis of sets that are homeomorphic to $\mathcal{C} \times(0,1)$.

Oversteegen has conjectured that this result generalizes to higher-dimensional solenoids [7], provided that the solenoid is homogeneous.

Conjecture 24. Suppose that an n-dimensional continuum $X$ is homogeneous. Then $X$ is homeomorphic to a solenoid if and only if $X$ has a 
basis of compact sets that are homeomorphic to $\mathcal{C} \times D_{n}$, the product of a Cantor set and an n-dimensional disk.

Spaces that have a local product structure $\mathcal{C} \times D_{n}$ often occur in fibrations over manifolds, as subsets that are transverse to the fiber. In this situation the conjecture is true.

Theorem 25. Suppose that $X$ is a homogeneous continuum and that $p: X \rightarrow M$ is a fibration over a closed manifold with zero-dimensional fiber and unique path lifting. Then $X$ is homeomorphic to a solenoid or a manifold.

Proof. Endow $M$ with a Riemannian metric $d$ and cover $M$ by a finite family of convex open sets $\mathcal{U}$. Endow $X$ with a metric $\widetilde{d}$ and let $\varepsilon>0$ be such that if $\widetilde{d}(x, y)<\varepsilon$ then $p(x)$ and $p(y)$ are contained in an element of $\mathcal{U}$. There exists an Effros $\delta$ for this $\varepsilon$, i.e., if $\widetilde{d}(x, y)<\delta$ then there is a homeomorphism $h: X \rightarrow X$ such that $h(x)=y$ and $\widetilde{d}\left(\operatorname{id}_{X}, h\right)<\varepsilon$.

Let $G$ be the group of fiber preserving homeomorphisms of $X$. Since $p$ has unique path lifting, an evaluation map of $G$ into a fiber is injective. Hence $G$ is a zero-dimensional group. Fix a fiber $\mathcal{F}=p^{-1}(m) \subset M$. Suppose that $x, y \in \mathcal{F}$ and that $\widetilde{d}(x, y)<\delta$. Since $\mathcal{U}$ is a convex cover and by the choice of $\delta$, there exists a homeomorphism such that $h(x)=y$ and $p \circ h$ is homotopic to the identity. Since $p$ is a fibration, $h$ is homotopic to a homeomorphism $\widetilde{h} \in G$ that necessarily maps $x$ to $y$. So $G \cdot x$ is an open subset of $\mathcal{F}$, which decomposes as a finite union of disjoint sets $\mathcal{F}=G \cdot x_{1} \cup \cdots \cup G \cdot x_{k}$. So each $G \cdot x$ is compact, which implies that $G$ is compact and zero-dimensional, and so is either a finite group or homeomorphic to the Cantor set. The quotient space $X / G$ is a finite covering space of $M$. The mapping $X \rightarrow X / G$ is a principal bundle over a manifold with either a finite structure group or a Cantor structure group, hence it is either a manifold or a solenoid.

Corollary 26. Suppose that $X$ is a homogeneous continuum with a local product structure $\mathcal{C} \times D_{n}$ and that $p: X \rightarrow M$ is a fibration over a closed $n$-dimensional manifold. Then $X$ is homeomorphic to a solenoid.

So Oversteegen's conjecture comes down to the problem of whether a homogeneous space with a local product structure $\mathcal{C} \times D_{n}$ fibers over a manifold. This is very similar to an old conjecture of Williams, that an expanding attractor fibers over a manifold. Williams's conjecture was eventually disproved by Farrell and Jones [6].

6. Remark. Hansen [9] has shown that a covering space over an $n$ dimensional manifold $M$ factors through $M \times \mathbb{R}^{n+1}$ (see also [18]). This is an embedding in the stable range which has been studied quite extensively 
in a general setting even beyond metric spaces [22]. If $f: X \rightarrow Y$ is a mapping with a $k$-dimensional fiber and $\operatorname{dim} Y=n$, then $f$ factors through the projection of $Y \times \mathbb{R}^{n+2 k+1}$ onto the first coordinate.

\section{References}

[1] J. M. Aarts, C. L. Hagopian and L. G. Oversteegen, The orientability of matchbox manifolds, Pacific J. Math. 150 (1991), 1-12

[2] G. Allaud and E. S. Thomas, Jr., Almost periodic minimal sets, J. Differential Equations 15 (1974), 158-171.

[3] A. Clark, A generalization of Hagopian's theorem and exponents, Topology Appl. 117 (2002), 273-283.

[4] A. Clark and R. J. Fokkink, Bihomogeneity of solenoids, Algebr. Geom. Topol. 2 (2002), 1-8.

[5] D. van Dantzig und B. L. van der Waerden, Über metrisch homogene Räume, Abh. Math. Sem. Hamburg 6 (1928), 367-376.

[6] F. T. Farrell and L. E. Jones, New attractors in hyperbolic dynamics, J. Differential Geom. 15 (1980), 107-133.

[7] R. J. Fokkink and L. G. Oversteegen, Homogeneous weak solenoids, Trans. Amer. Math. Soc. 354 (2002), 3743-3755.

[8] B. Günther and J. Segal, Every attractor of a flow on a manifold has the shape of a finite polyhedron, Proc. Amer. Math. Soc. 119 (1993), 321-329.

[9] V. L. Hansen, Braids and Coverings, Selected Topics, London Math. Soc. Student Texts 18, Cambridge Univ. Press, Cambridge, 1989.

[10] G. Hector and U. Hirsch, Introduction to the Geometry of Foliations. Part A, Aspects Math., Vieweg, Braunschweig, 1981.

[11] J. Keesling and D. C. Wilson, Embedding $T^{n}$-like continua in Euclidean space, Topology Appl. 21 (1985), 241-249.

[12] K. Kuperberg, On the bihomogeneity problem of Knaster, Trans. Amer. Math. Soc. 321 (1990), 129-143.

[13] —, Bihomogeneity and Menger manifolds, Proc. Internat. Conf. on Set-theoretic Topology and its Applications, Part 2 (Matsuyama, 1994), Topology Appl. 84 (1998), 175-184.

[14] M. C. McCord, Inverse limit sequences with covering maps, Trans. Amer. Math. Soc. 114 (1965), 197-209.

[15] -, Embedding P-like compacta in manifolds, Canad. J. Math. 19 (1967), 321-332.

[16] J. van Mill, Infinite-Dimensional Topology. Prerequisites and Introduction, NorthHolland, Amsterdam, 1989.

[17] -, A new proof of Effros' theorem, preprint, Free University, Amsterdam, 2001.

[18] P. V. Petersen, Fatness of covers, J. Reine Angew. Math. 403 (1990), 154-165.

[19] R. Sacksteder, On the existence of exceptional leaves in foliations of co-dimension one, Ann. Inst. Fourier (Grenoble) 14 (1964), no. 2, 221-225.

[20] R. Schori, Inverse limits and homogeneity, Trans. Amer. Math. Soc. 124 (1966), 533-539.

[21] E. S. Thomas, Jr., One-dimensional minimal sets, Topology 12 (1973), 233-242.

[22] H. M. Tuncali and V. Valov, On finite-dimensional maps, arxiv, math.GN/0209074.

[23] J. de Vries, Elements of Topological Dynamics, Math. Appl. 257, Kluwer, Dordrecht, 1993. 
[24] R. F. Williams, Expanding attractors, Inst. Hautes Études Sci. Publ. Math. 43 (1974), 169-203.

[25] T. Yagasaki, Homotopy types of homeomorphism groups of noncompact 2-manifolds, Topology Appl. 108 (2000), 123-136.

Department of Mathematics

University of North Texas

Denton TX 76203-1430, U.S.A.

E-mail: AlexC@unt.edu
Faculty of Information Technology and Systems Technische Universiteit Delft P.O. Box 5031 2600 GA Delft, Netherlands E-mail: r.j.fokkink@its.tudelft.nl

Received 1 October 2002;

in revised form 25 January 2004 